\title{
A true vascular aneurysm of the hepatic artery proper as a rare cause of nonmalignant painless jaundice
}

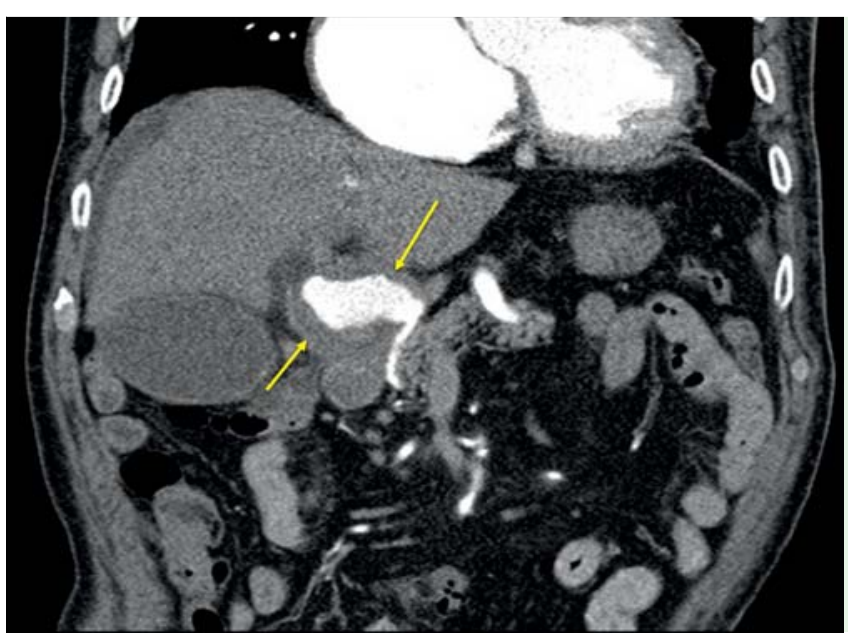

Fig. 1 The partially thrombotic aneurysm of the hepatic artery proper (yellow arrows) extended from the junction of the gastroduodenal artery up to the branching of the right and left hepatic artery (diameter $4.3 \mathrm{~cm}$ ).

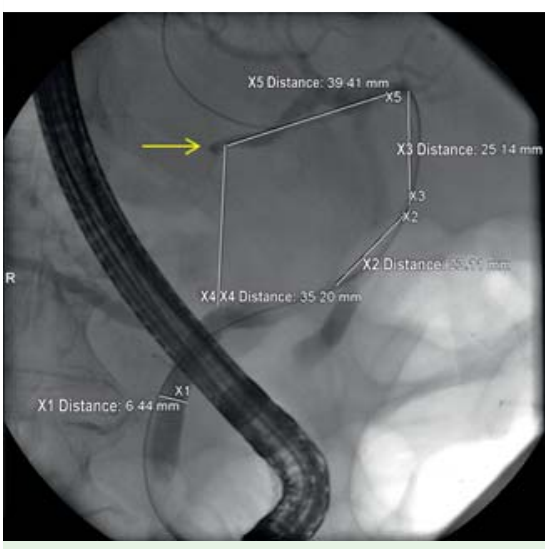

Fig. 3 At endoscopic retrograde cholangiopancreatography, the area without contrast media filling was measured as $39 \times 47 \mathrm{~mm}$, corresponding to the aneurysm of the hepatic artery proper, which compressed the biliary duct and left-sided segment branches (yellow arrow).

Rare causes of painless jaundice include parasitic infections and lymphoma. To date, two cases of vascular pseudoaneurysm in acute cholecystitis and chronic pancreatitis have been reported [1,2].

An 85-year-old man was diagnosed by contrast-enhanced computed tomography scan with a partially thrombotic aneurysm of the hepatic artery proper, which was compressing the common bile duct (CBD) (৫ Fig. 1). An initial attempt to place an endoprosthesis via endoscopic retrograde cholangiopancreatography (ERCP) failed, and obstructive cholangitis developed (bilirubin $23.1 \mathrm{mg} / \mathrm{dL}, \mathrm{C}$-reactive protein

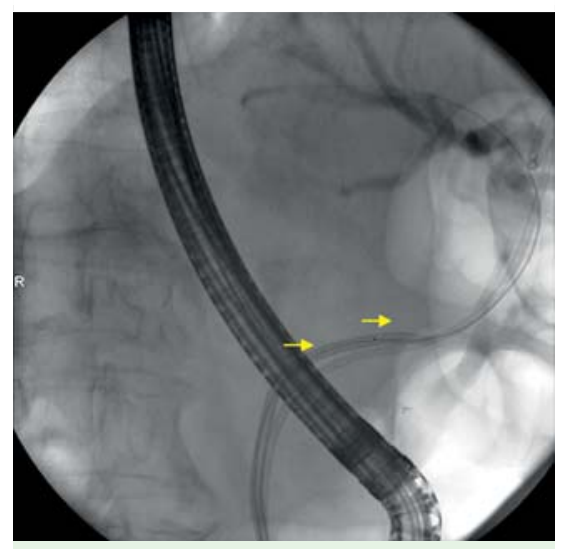

Fig. 4 An initial 7-Fr double-pigtail endoprosthesis (yellow arrows) was inserted into the biliary hilus and the right segment VII to serve as guide for the second endoprosthesis, which had to be inserted carefully around and over the aneurysm into the left-sided dilated segments.

$116 \mathrm{mg} / \mathrm{L}$, leukocytosis $17800 / \mu \mathrm{L})$, which required antibiotic treatment, resection, and/or a second problem-focused ERCP.

Resection was discussed but was not considered to be feasible due to significant cardiovascular co-morbidity. Therefore, biliary tract decompression by ERCP was planned.

ERCP was particularly challenging. At a distance of $35 \mathrm{~mm}$ from the papilla, below the junction of the cystic duct, the vascular aneurysm caused a moderately severe smooth-walled stenosis (50\%-90\%), measuring at least $45 \mathrm{~mm}$ in length. The external compression resulted in a curved

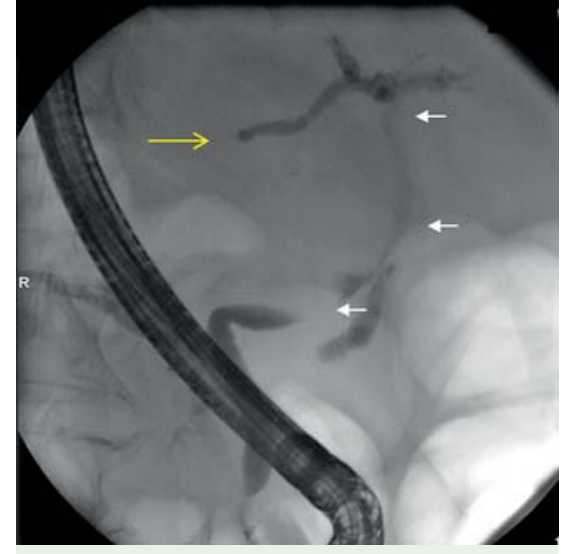

Fig. 2 At endoscopic retrograde cholangiopancreatography, the distal bile duct showed normal width, filling, and bile duct wall. However, approximately $35 \mathrm{~mm}$ above the papilla, the bile duct was bent at right angles and showed a 45-mm long stenosis in the middle and upper parts, extending upwards to the biliary hilus (white arrows). Of note, the descending biliary branch of the liver segment III also showed a termination of the duct (yellow arrow).

CBD with a right-angled kink $(\bullet$ Fig. 2 and - Fig.3). After endoscopic papillotomy, widening of the stenosis was achieved by careful use of bougies (5-10 Fr). Subsequently, one double-pigtail endoprosthesis was placed in the right hepatic duct $(7 \mathrm{Fr} / 16 \mathrm{~cm})$ to serve as a splint for the second endoprosthesis, which had to be implanted around and over the aneurysm to finally reach the dilated biliary ducts of the left liver segments $(10 \mathrm{Fr} / 12 \mathrm{~cm}$; $\checkmark$ Fig. 4 and $\bullet$ Fig.5). Correct stent placement was confirmed by postinterventional ultrasound ( $\bullet$ Fig. 6 ).

Interventional occlusion of the aneurysm was not performed due to the risk of wide-ranging ischemia. Thus, only mechanical biliary drainage evidenced by decreasing cholestasis was able to circumvent the complications of this rare vascular cause of bile duct compression.

In contrast to arterial pseudoaneurysms, which are a rare but established complication of ERCP $[3,4]$, this is, to our knowledge, the first case of a true vascular aneurysm leading to progressive cholangitis that required treatment by ERCP. 


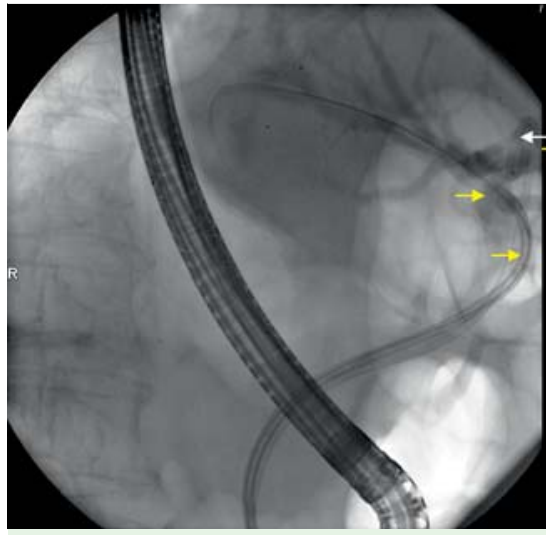

Fig.5 Insertion of the second 10-Fr endoprosthesis (yellow arrows) over the right-angled corner into the liver segment III (white arrow) for definitive drainage of the left liver.

Endoscopy_UCTN_Code_CCL_1AZ_2AN

Competing interests: None

\section{Martin Raithel ${ }^{1}$, Ingo Ganzleben', Jürgen Gschossmann², Alexander F. Hagel ${ }^{1}$, Markus F. Neurath ${ }^{1}$, Ruediger S. Goertz ${ }^{1}$}

${ }^{1}$ Department of Medicine 1, University of Erlangen-Nuremberg, Erlangen, Germany

${ }^{2}$ Department of Internal Medicine, Klinikum Forchheim, Forchheim, Germany

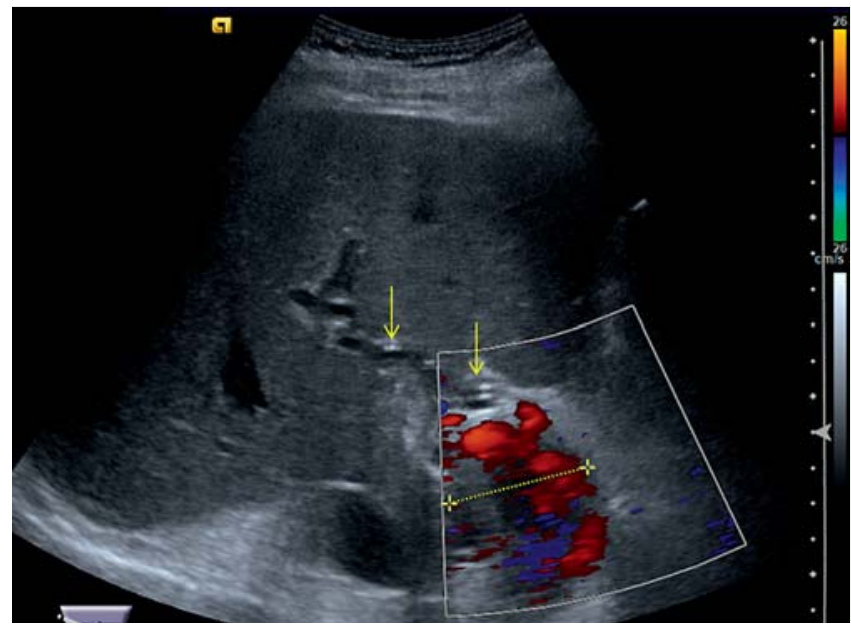

Fig. 6 Intercostal plane after stenting (stent indicated by yellow arrows), showing the partially thrombotic aneurysm of the hepatic artery proper compressing the main bile duct. Absence of significant intrahepatic cholestasis can be appreciated. Measurement of aneurysm: $4.0 \mathrm{~cm}$ (yellow line).

\section{References}

1 Anwar Z, Sayani R, Kanwal D et al. Hepatic artery pseudoaneurysm mimicking Mirizzi syndrome. J Coll Physicians Surg Pak 2013; 23: 504-506

2 Becheur H, Zins M, Levy P et al. [A rare cause of obstructive jaundice: peripancreatic pseudoaneurysm]. Gastroenterol Clin Biol 1996; 20: 1131 - 1134

3 Gaduputi V, Tariq H, Dev A. Visceral arterial aneurysms complicating endoscopic retrograde cholangiopancreatography. Case Rep Gastrointest Med 2013; 2013: 515201

4 Asayama $N$, Sasaki T, Serikawa $M$ et al. [Hepatic artery pseudoaneurysm after endoscopic biliary stenting for pancreatic cancer]. Nihon Shokakibyo Gakkai Zasshi 2014; 111: 931 - 939
Bibliography

DOI http://dx.doi.org/

10.1055/s-0034-1390846

Endoscopy 2014; 46: E652-E653

(c) Georg Thieme Verlag KG

Stuttgart · New York

ISSN 0013-726X

Corresponding author

\section{Martin Raithel, MD}

Department of Medicine 1

University of Erlangen-Nuremberg

Ulmenweg 18

91054 Erlangen

Germany

Phone: +49-9131-8535000

martin.raithel@uk-erlangen.de 